\title{
„Are we doing alright?“ Die Komplexität ethisch-verantwortlicher Forschung zu sexueller Orientierung, geschlechtlicher Identität und Gesundheit im südlichen und östlichen Afrika
}

\author{
Alex Müller \\ Angenommen: 25. März 2021 / Online publiziert: 15. April 2021 \\ (C) Der/die Autor(en) 2021
}

\section{Einführung}

Sexuelle Orientierung und geschlechtliche Identität sind soziale Determinanten der Gesundheit. Stigmatisierung, Diskriminierung und soziale Ausgrenzung aufgrund von heteropatriarchalen Normen und Vorurteilen haben negative Auswirkungen auf die Gesundheit von lesbischen, schwulen, bisexuellen, queeren und trans (LSBQT+) Menschen. Gesundheitswissenschaftliche Studien zeigen, dass LSBQT+ Menschen, im Vergleich zu cisgender und heterosexuellen Menschen, häufiger jünger sterben, häufiger an Depressionen oder Angstzuständen leiden und ein erhöhtes Risiko für Übergewichtigkeit, Herz-Kreislauf-Erkrankungen und Krebserkrankungen haben (Logie 2012; Pega und Veale 2015).

Die Pathologisierung von sexueller und geschlechtlicher Vielfalt durch gesundheitswissenschaftliche Forschung und Praxis hat den medizinisch-rechtlichen Diskurs und die öffentliche Meinung nachhaltig geprägt (Drescher 2010). Im Gegenzug können Ansätze, die sexuelle Orientierung und geschlechtliche Identität als soziale Determinanten der Gesundheit betonen, nun dazu beitragen, durch wissenschaftliche Evidenz zur rechtlichen und sozialen Gleichstellung und Inklusion von LSBQT+ Menschen beizutragen. Diese Ansätze bewegen sich jedoch in einem gesellschaftlichen Spannungsfeld. Unterstützt gesundheitswissenschaftliche Forschung in solch politisierten Umfeldern stets Bemühungen zur Verbesserung der Lebensbedingungen von marginalisierten und illegalisierten Menschen? Oder trägt sie auch zu einer vermehrten negativen öffentlichen Sichtbarkeit und einer verstärkten Stigmatisierung bei?

Prof. Dr. med. A. Müller ( $\square)$

Institut für Ethik und Geschichte der Medizin, Universitätsmedizin Göttingen,

Humboldtallee 36, 37073 Göttingen, Deutschland

E-Mail: alexandra.mueller@med.uni-goettingen.de 
In diesem Beitrag analysiere ich die Komplexität von ethisch-verantwortlicher Forschung zu sexueller Orientierung und geschlechtlicher Identität anhand einer Studie, die ich zusammen LSBQT+ Menschen im südlichen und östlichen Afrika durchgeführt habe, wo sexuelle und geschlechtliche Vielfalt rechtlich und soziopolitisch umstritten sind. An diesem konkreten Beispiel zeige ich Wege auf, mit forschungsethischen Risiken in politisch brisanten Kontexten verantwortlich umzugehen.

\section{Die Studie: „Are we doing alright?““}

Als Leiterin einer Forschungsgruppe zu Fragen von sexueller Orientierung, Geschlechtsidentität und Gesundheit an der Universität Kapstadt wurde ich im Oktober 2015 zu einem Treffen mit Nichtregierungsorganisationen aus Ländern des südlichen und östlichen Afrikas eingeladen. Das Treffen wurde auf Initiative einer niederländischen Stiftung organisiert, nachdem die Organisationen wiederholt auf die unzureichende Datenlage zur Gesundheit von LSBQT+ Menschen in ihren Ländern hingewiesen und gefordert hatten, die Auswirkungen von Stigma und Ausgrenzung von LSBQT+ Menschen wissenschaftlich zu untersuchen. Aus diesem Treffen ging das Southern and East African Research Collective on Health (SEARCH) hervor: ein Netzwerk bestehend aus 23 Organisationen aus Äthiopien, Botswana, Eswatini, Kenia, Lesotho, Malawi, Südafrika, Sambia und Simbabwe, der niederländischen Stiftung sowie meiner Forschungsgruppe. Mit der Studie „Are we doing alright?“ befragten wir zwischen 2016 und 2018 fast 4000 LSBQT+ Menschen in den neun Ländern. Aus dem Wirkungskreis der beteiligten Partnerorganisationen gewannen wir Teilnehmende, die entweder in den Büros der Organisationen, an einem Ort ihrer Wahl, oder online mit Hilfe eines Fragebogens befragt wurden. Unsere Ergebnisse zeigen, dass Gewalterfahrungen unter LSBQT+ Menschen weiter verbreitet sind als in der Gesamtbevölkerung; dass LSBQT+ Menschen häufiger als die Gesamtbevölkerung an Depressionen, Angstzuständen und Suizidalität leiden, und dass bis zu einem Drittel der befragten LSBQT+ Menschen der Zugang zur gesundheitlichen Versorgung aufgrund ihrer sexuellen Orientierung und Geschlechtsidentität verwehrt wurde (Müller et al. 2021, 2019).

Mit Ausnahme von Lesotho und Südafrika (und seit 2019 Botswana) steht gleichgeschlechtliche Sexualität in diesen Ländern unter Strafe. Während der Wortlaut des aus der britischen Kolonialzeit stammenden Gesetzes in den einzelnen Ländern nahezu identisch ist, variiert dessen Umsetzung von einem de-facto Moratorium bis hin zu aktiver Strafverfolgung. Eine Verurteilung wegen gleichgeschlechtlicher Handlungen kann Haftstrafen von bis zu 35 Jahren nach sich ziehen. Dies erzeugt ein soziopolitisches Klima, in dem Ausgrenzung, Diskriminierung und Gewalt gegen LSBQT+ Menschen im besten Fall nicht geahndet und im schlimmsten Fall gefördert werden. Obgleich die Gesundheitsversorgung und -forschung mit LSBQT+ Menschen rechtlich nicht verboten ist, trägt dieses Umfeld von Homo- und Transphobie dazu bei, dass Beschäftigte im Gesundheitswesen die Versorgung von LSBQT+ Patienten verweigern, LSBQT+ Patienten während der Behandlung diskriminieren oder im schlimmsten Fall den Behörden melden (Müller et al. 2018). 
Für die Durchführung unserer Studie stellten staatliche Repression und soziale Stigmatisierung daher signifikante Risiken dar. Forschungsteilnehmende riskieren die Aufdeckung ihrer sexuellen Orientierung oder Geschlechtsidentität, etwa wenn Studiendokumente (z. B. Aufklärungsbögen) bei ihnen gefunden werden, oder wenn staatliche Sicherheitskräfte Daten von Teilnehmenden im Rahmen von Razzien bei Organisationen beschlagnahmen. Auch für Forschende und Datenerhebende ist die Durchführung einer solchen Studie nicht ungefährlich, da die Verbindung zu LSBQT+-assoziierten Themen je nach politischer Lage eine sensationalisierte Medienberichterstattung, Drohungen oder auch Repressionen nach sich ziehen kann. Hinzu kommt, dass Themen von sexueller und geschlechtlicher Vielfalt oftmals für politische Zwecke instrumentalisiert werden. Konkret besteht die Gefahr, dass Forschungsergebnisse absichtlich fehlinterpretiert werden, um bestimmte homo- und transphobe Entscheidungen politisch zu rechtfertigen. So wird das Verbot von gleichgeschlechtlichem Sex zum Beispiel mit der Verhinderung von HIV-Infektionen begründet, da schwule Männer ein erhöhtes HIV-Infektionsrisiko hätten.

Im Folgenden stelle ich wichtige ethische und praktische Erwägungen im Zusammenhang mit diesen Forschungsrisiken vor und zeige auf, wie Studien zu sexueller Orientierung, Geschlechtsidentität und Gesundheit zu Gleichstellungsbemühungen beitragen können.

\section{Partizipatives Arbeiten}

Im Rahmen der Studie arbeiteten wir, gemäß existierender Richtlinien von Nichtregierungsorganisationen zur Arbeit mit LSBQT+ Menschen in restriktiven rechtlichen Umfeldern (KP Reach 2018), partizipativ als gleichberechtigte Entscheidungstragende, d.h. sowohl Forschungsfragen und Methodik als auch Ansätze zur Datenanalyse und Strategien zur Publikation und Dissemination wurden gemeinsam von allen 23 Organisationen und meiner Forschungsgruppe entschieden. Dieser Ansatz stellte zum einen sicher, dass die erhobenen Daten für die weitere Arbeit der Organisationen von Bedeutung waren; zum anderen konnten wir auf diese Weise unser Vorgehen an die jeweiligen spezifischen soziopolitischen und rechtlichen Umstände anpassen und auf sich rasch ändernde politischen Umstände schnell und gut informiert reagieren. Dies trug zur Minimierung der Sicherheitsrisiken für Teilnehmende, Datenerhebende und Forschende bei.

\section{Ethische Begutachtung und Bewilligung}

Unsere Studie wurde von der Ethikkommission der gesundheitswissenschaftlichen Fakultät der Universität Kapstadt genehmigt. Weiterhin beantragten wir Genehmigungen von Ethikkommissionen oder Gesundheitsbehörden der einzelnen Länder, in denen wir Befragungen durchgeführten. Die Einhaltung dieses forschungsethischen Standards ist jedoch in Ländern, in denen sexuelle und geschlechtliche Minderheiten erhöhter staatlicher Repression ausgesetzt sind, mit besonderen Risiken verbunden, die es abzuwägen galt. Tatsächlich hätte sich in solchen Ländern das Sicherheitsrisiko für unsere Partnerorganisationen und Forschungsteilnehmenden erheblich erhöht, da ein Ethikantrag ihre Existenz explizit gemacht und staatliche Behörden damit zur 
Abkehr von der bislang üblichen Haltung des „Don't ask, don't tell“ hin zu einer offiziellen Anerkennung dieser Organisationen und an der Studie teilnehmenden LSBQT+ Menschen gezwungen hätte. In diesen Ländern initiierten wir stattdessen einen „Community Review“-Prozess, in dem lokale LSBQT+ Menschen und Akademiker, die mit Forschungsethik und der soziopolitischen Situation in dem jeweiligen Land vertraut waren, unser Studienprotokoll bewerteten und ihre Empfehlung abgaben (siehe auch Amon et al. 2012).

\section{Vorsorge und Flexibilität während der Datenerhebung}

Die Daten wurden durch die lokalen Organisationen selbst erhoben, nachdem meine Forschungsgruppe alle Beteiligten in Forschungsmethodik und Ethik geschult hatte. Die Datenerhebung erfolgte schrittweise, für jeweils drei Monate in den ausgewählten Ländern. Begonnen wurde mit Ländern, in denen die rechtliche und politische Situation von LSBQT+ Menschen für Studienteilnehmende das geringste Risiko darstellte. Jede Organisation hatte im Vorfeld ein umfassendes Sicherheitsprotokoll vor dem Hintergrund der jeweiligen soziopolitischen Situation erstellt, sowie sichere Anlaufstellen für rechtliche Beratung und psychosoziale Betreuung von Teilnehmenden identifiziert. Aufgrund der soziopolitischen Einschätzungen wurden für die Durchführung der Studie auf das jeweilige Land zugeschnittene Sicherheitsvorkehrungen getroffen. So gab es für Teilnehmende zum Beispiel die Möglichkeit, ihre Einwilligung lediglich durch Ankreuzen zu geben, um eine etwaige Identifizierung durch ihre Unterschrift zu verhindern. Fragebögen waren nicht nur auf Papier, sondern auch online verfügbar, damit Datenerhebende nicht gezwungen waren LSBQT+assoziiertes Material mit sich zu führen. Onlinedaten wurden über die verschlüsselte Plattform RedCap erhoben und Teilnehmende über Datenschutzmaßnahmen eingehend informiert. Ferner verfügten wir im Rahmen der Studie über ein Budget zur Soforthilfe im Falle von Verhaftungen oder anderen Notfällen und hielten ständigen Kontakt zu den Datenerhebenden über eine WhatsApp-Gruppe, um rasch reagieren zu können. Auch waren wir gezwungen, die Datenerhebungszeiträume an politische Ereignisse anzupassen, da Politikerinnen und Politiker z. B. zur Stärkung ihrer Position vor Wahlen verstärkt auf öffentliche Homo- und Transphobie setzen. Als staatliche Repression und Medienaufmerksamkeit eine sichere Datenerhebung in einigen Ländern vorübergehend unmöglich machten, unterbrachen wir die Studie für einige Monate zur Sicherheit für alle Beteiligten.

\section{Gemeinsame Nutzung von Forschungsdaten: sozial engagierte Forschung}

Die länderspezifischen Datensätze stehen den Partnerorganisationen der jeweiligen Länder zur freien Verfügung. Die Verwendung von Forschungsdaten für wissenschaftliche Publikationen und zur Interessensvertretung wurde - und wird weiterhin - in Absprache mit allen Beteiligten entschieden. Ideen für wissenschaftliche Publikationen werden allen Partnerorganisationen vorgestellt und die Mitarbeit daran ist offen für alle, die an der Verfassung eines Beitrags mitwirken möchte. Des Weiteren ist es ein grundlegendes Prinzip, dass Veröffentlichungen nicht nur in wissenschaftlichen Fachzeitschriften erscheinen, sondern auch für spezifische Anläs- 
se zum Zweck der Interessensvertretung auf staatlicher oder internationaler Ebene verfasst werden. Hierbei treffen wir Entscheidungen über die Autorenschaft nach strategischen Überlegungen, die berücksichtigen, wie die Glaubwürdigkeit der vorzustellenden Ergebnisse beeinflusst sein könnte. So entschieden wir zum Beispiel, dass eine Präsentation der Ergebnisse bei der African Commission for Human and People's Rights von mir als universitätsassoziierter Wissenschaftlerin gehalten werden sollte, da dies die regelgerechte Durchführung der Studie und die Objektivität der Daten am besten widerspiegele. Für einen Vortrag in einem staatlichen Gesundheitsministerium wurde hingegen eine der lokalen Partnerorganisationen ausgewählt, da dieser nicht unterstellt werden konnte, sich von außen in die inneren Angelegenheiten des Landes einzumischen.

Am 11. Juni 2019 entschied der Oberste Gerichtshof Botswanas, dass die Paragraphen 164, 167 und 168 des Strafgesetzbuchs, welche gleichgeschlechtliche Sexualität unter Strafe stellen, mit der auf demokratischen Rechten basierenden Verfassung des Landes unvereinbar seien. Damit wurde gleichgeschlechtliche Sexualität de facto entkriminalisiert. Einer der Beweise, auf das sich das bahnbrechende Urteil stützte, waren Forschungsdaten zu den negativen Auswirkungen des Gesetzes auf die Gesundheit von LSBQT+ Menschen. Die Daten stammten aus der Studie ,Are we doing alright?"“. Ich hatte sie in Form einer eidesstattlichen Expertise während der Beweisaufnahme in das Gerichtsverfahren eingebracht, nachdem dies als Strategie von den Partnerorganisationen aus Botswana vereinbart worden war. Dieses Beispiel belegt das Potential gesundheitswissenschaftlicher Forschung, zur Verbesserung der rechtlichen (und soziopolitischen) Rahmenbedingungen von marginalisierten und kriminalisierten Gruppen beizutragen.

\section{Ausblick}

Zum Spannungsfeld zwischen Risiken und Nutzen gesundheitswissenschaftlicher Forschung mit marginalisierten und illegalisierten Menschen im südlichen und östlichen Afrika gibt es durchaus Parallelen in aktueller Forschung in Deutschland. So sind die aufgezeigten ethisch komplexen Risiken der Sichtbarmachung von LSBQT+ Menschen übertragbar auf Forschung mit Menschen ohne offiziellen Aufenthaltsstatus, Sexarbeitenden, Drogenkonsumierenden, oder Menschen, die für Schwangerschaftsabbrüche nach Deutschland reisen, da diese im eigenen Land illegal sind. Auch hier ergibt sich die Notwendigkeit partizipativer und sozial engagierter Forschungsansätze, um der vollen Komplexität ethisch-verantwortlicher Forschung gerecht zu werden.

\section{Notiz}

Die Mitglieder des Southern and East African Research Collective on Health sind: BONELA, Lesbians, Gays and Bisexuals of Botswana, Rainbow Identity Association und Dr Chelsea Morroni (Botswana); Rock of Hope und Sindy Matse (Eswatini); The People's Matrix Organisation (Lesotho); Ishtar-MSM, Jintsiangu, Maaygo, Minority Women in Action, National Gay and Lesbian Human Rights Commission, People 
Marginalized and Aggrieved (Kenya); Centre for the Development of People und Prof Adamson Muula (Malawi); COC Nederland (Niederlande); Friends of Rainka, The Lotus Identity, Transbantu Zambia (Sambia); GALZ - An LGBTI Association in Zimbabwe, Sexual Rights Centre und Dr Nelson Muparamoto (Simbabwe); Durban Lesbian and Gay Centre, Gender Dynamix, OUT LGBT Wellbeing, Triangle Project und das Team der Gender Health and Justice Research Unit (Südafrika); sowie zwei ungenannte Organisationen in Äthiopien.

Funding Open Access funding enabled and organized by Projekt DEAL.

Open Access Dieser Artikel wird unter der Creative Commons Namensnennung 4.0 International Lizenz veröffentlicht, welche die Nutzung, Vervielfältigung, Bearbeitung, Verbreitung und Wiedergabe in jeglichem Medium und Format erlaubt, sofern Sie den/die ursprünglichen Autor(en) und die Quelle ordnungsgemäß nennen, einen Link zur Creative Commons Lizenz beifügen und angeben, ob Änderungen vorgenommen wurden.

Die in diesem Artikel enthaltenen Bilder und sonstiges Drittmaterial unterliegen ebenfalls der genannten Creative Commons Lizenz, sofern sich aus der Abbildungslegende nichts anderes ergibt. Sofern das betreffende Material nicht unter der genannten Creative Commons Lizenz steht und die betreffende Handlung nicht nach gesetzlichen Vorschriften erlaubt ist, ist für die oben aufgeführten Weiterverwendungen des Materials die Einwilligung des jeweiligen Rechteinhabers einzuholen.

Weitere Details zur Lizenz entnehmen Sie bitte der Lizenzinformation auf http://creativecommons.org/ licenses/by/4.0/deed.de.

\section{Einhaltung ethischer Richtlinien}

Interessenkonflikt Alex Müller gibt an, dass kein Interessenkonflikt besteht.

Ethische Standards Für diesen Beitrag wurden von der Autorin keine Studien an Menschen oder Tieren durchgeführt. Für die aufgeführten Studien gelten die jeweils dort angegebenen ethischen Richtlinien.

\section{Literatur}

Amon JJ, Baral SD, Beyrer C, Kass NE (2012) Human rights research and ethics review: protecting individuals or protecting the state? PLoS Med 9:e1001325

Drescher J (2010) Queer diagnoses: parallels and contrasts in the history of homosexuality, gender variance, and the diagnostic and statistical manual. Arch Sex Behav 39:427-460. https://doi.org/10.1007/ s10508-009-9531-5

KP Reach (2018) Guidelines for conducting participatory social research with key populations and marginalised communities. http://kpreach.net/category/guidelines/. Zugegriffen: 12. März 2021

Logie C (2012) The case for the World Health Organization's Commission on the Social Determinants of Health to address sexual orientation. Am J Public Health 102:1243-1246. https://doi.org/10.2105/ AJPH.2011.300599

Müller A, Daskilewicz K, Kabwe ML, Mmolai-Chalmers A, Morroni C, Muparamoto N, Southern and East African Research Collective on Health (2021) Experience of and factors associated with violence against sexual and gender minorities in nine African countries: a cross-sectional study. Bmc Public Health 21:357

Müller A, Daskilewicz K, Southern and East African Research Collective on Health (2019) Are we doing alright? Realities of violence, mental health, and access to healthcare related to sexual orientation and gender identity and expression in East and Southern Africa: research report based on a communityled study in nine countries. Gender, Health and Justice Research Unit, Cape Town 
Müller A, Spencer S, Meer T, Daskilewicz K (2018) The no-go zone: a qualitative study of access to sexual and reproductive health services for sexual and gender minority adolescents in Southern Africa. Reprod Health 15:12. https://doi.org/10.1186/s12978-018-0462-2

Pega F, Veale JF (2015) The case for the World Health Organization's Commission on Social Determinants of Health to address gender identity. Am J Public Health 105:e58-e62. https://doi.org/10.2105/AJPH. 2014.302373 